\title{
A Survey of Cell Population Dynamics
}

\author{
O. ARINO ${ }^{\mathrm{a}}$ and E. SÁNCHEZ ${ }^{\text {b.* }}$
}

${ }^{a}$ Laboratoire de Mathématiques Appliquées, I.P.R.A. Université de Pau. 64000 Pau. France; ${ }^{b}$ Dpto Mathemáticas, E.T.S.I. Industriales, U.P.M., C/José Gutiérrez Abascal, 2, 28006 Madrid, Spain

\begin{abstract}
A survey of cell-population models is presented. The so-called cell cycle is discussed, including some models which are not population models but which contribute to the better understanding proliferation of cell dynamics. Classical linear models of structured cell-population models are described with a review of classical results and a comparison between different approaches. Finally, some non-linear models are also developed.
\end{abstract}

Key'words: Cell kinetics, cell cycle, structured population dynamics

\section{INTRODUCTION: THE CELL CYCLE}

Cell life spans from the birth of a cell as a result of the division of another cell, the mother cell, into two, the daughter cells, to the moment when this cell has completed its division into two new cells. There are cells which never divide or are destroyed before they divide. In normal conditions, cells of a given line tend to follow the same path. The life of an ordinary cell passes through a sequence of recognizable transitions, which repeats itself to cells of the next generation. This series of events is known as the 'cell cycle.'

The best known scenario for the description of the cell cycle considers four successive periods G1, S, G2, M. Cells enter the cell cycle in G1. This phase does not seem to have a distinctive feature, in fact, the letter $\mathrm{G}$ refers to the word 'gap', i.e. the name given to a non-distinctive part of the cycle. They then enter a phase of active production, the DNA synthesis, known as the S-phase. This phase can be empirically determined. It is followed by another, not easily distinguishable period, the G2 phase. Finally, the cell starts the process which ends up with the formation of two new cells, known as the mitosis or M-phase.

During its whole life, the cell grows in size, not only because it doubles its DNA content, but also in other constituents such as RNA. It is commonly admitted that the size is closely related to the mechanisms regulating cell life (Mitchinson, 1971; John, 1981) but that size does not refer to a unique and well-defined character of a cell. It can be the mass of some constituent such as RNA or even a hypothetical constituent, a mitogen in the sense of Lasota and Mackey (1984).

The research reported in this paper was first presented at the year long workshop on Mathematics in Medicine held at the International Centre for Mathematical Sciences, Edinburgh during 1994-1995.

${ }^{*}$ Corresponding Author: E-mail: ESANCHEZ@ETSII.UPM.ES. 


\section{Molecular Biology Approach to the Cell Cycle}

The cell cycle has been investigated for many years at the physiological level and recently by molecular biological methods. Starting at the end of the 1980s, cell dynamics modeling developed in a new direction. Structured cell population models had been pushed to a limit, well illustrated by the general model analyzed in Rossa (1995). Experimental findings from cell molecular biology gave a strong impetus to the search for models describing some of the biochemical mechanisms underlying cell development processes. We briefly comment on this.

First of all, we want to underline that the study of cells at the molecular level is not exactly recent. Many of the results used today were discovered in the 1980s. As an example, Nurse and Bisset (1981) report that, in yeast, $c d c 2$ and $c d c 10$ genes are both needed for completion of G1 ( $c d c$ means cell division cycle). Nurse and his collaborators identified several other genetic elements throughout the 1980s (Forsburg and Nurse, 1991).

One of the puzzling facts regarding the size structured models which were so popular in the 1980s is the case of embryonic cells. On the one hand, embryonic cells do not grow in size. They undergo a series of rapid division cycles in the absence of any growth. On the other hand, these divisions do not seem to be regulated by doubling of nuclear content (DNA) since anucleate embryonic cells continue to divide (Harvey, 1940). It became more and more evident that size growth is not always a faithful indicator of the progress through the cell cycle and that molecular biology data should be taken into account.

In this context cell cycle is explained via the biochemical reactions which occur within each cell. It is accepted that the evolution through different steps of the cell cycle is due to fluctuations in concentrations of some proteins (cyclins) and this mechanism is essentially the same for all types of cells. Roughly speaking, cyclins A and B are produced at a constant rate in the cell and their concentrations change periodically along the cell cycle. Cyclin B is related to the production of another protein called 'maturation promotion factor' (MPF) which at high concentration gives rise to the mitotic phase (Hyver and Le
Guyader, 1990; Norel and Agur, 1991). The first models of this type concentrated only on this aspect. Focusing the study on a few biochemical species, supposedly the main ones, they aimed at describing the periodic oscillations of the concentrations (or of the quantities) of these products in a cell. To mention a few examples, in Thron (1991) and Norel and Agur (1991), a system of two first-order ODE (ordinary differential equation) satisfied by cyclin $\mathrm{B}$ and MPF is proposed. Numerical results indicate the existence of limit cycles. A more complex model involving six equations was introduced by Obeyesekere et al. (1992, 1994, 1995). State variables are concentrations of different product which control the so-called 'mitotic clock'.

It seems, however, that a correct description of cell dynamics, even restricted to the level of biochemical species present in the cell, has to take cellpopulation processes into account: both cell growth in size and division. Clearly, the cell-cycle clock is not a function of only concentration. Otherwise, daughter cells would have the same capacity of division as the mother cell and could divide right after, which would lead to smaller and smaller cells. This is not observed in normal conditions in nonembryonic cells. Division is also playing a role in the clock, by resetting the values of the species.

A model coupling cell growth and evolution at the molecular level was first proposed by Tyson (1991). An extension of the Tyson model is given by Novak and Tyson (1995). Roughly speaking, the model described by Novak and Tyson (1995) is composed of two subsystems. One is a two-dimensional system of ODE governing the evolution of two concentrations of the biochemical species. The functions defining the system are non-linear and depend on parameters which, in fact, are time-dependent and are solutions to the other subsystem. Amongst these parameters is the total mass of the cell. One can think of the variation of the second group of variables as rapid with respect to the concentrations so that they remain nearly constant while the cell progresses through the cycle. The qualitative feature of the main system (the one verified by the concentration) depends upon the values of the parameters 
or, more generally, the region where the parameters live. Tyo main modes can be depicted: the spontaneous oscillator mode, observed in embryonic cells, where rapid autonomous cycles repeat themselves, and the excitable switch mode, followed by nonembryonic cells in which cells can be switched from quiescence to proliferating state by some perturbation. A quasi-static model of excitable switch is described in Thron (in preparation).

Val and Tyson (in preparation) present a model for the budding yeast (Sacchoromyces cerevisae) combining a description of the cell cycle in terms of some molecular events, and a stage structured model is proposed. This structured model is a transport equation of the type described by Metz and Diekmann (1986) and Arino (1995). The vector of state is a double infinite sequence of functions $\left\{n_{j}^{0}(t, x)\right\},\left\{n_{j}^{1}(t, \tau, x)\right\}$.

$n_{j}^{0}$ is the density (with respect to $x$ ) of cells with $j$ scars, in Gl, called pre-START phase by Val and Tyson (in preparation); $n_{j}^{1}$ is the corresponding density of cells further on in the cell cycle (called post-START phase); $t$ is the chronological time, while $\tau$ is the time spent in post-START at time $t$. The position of a cell in the cycle is described by the vector $x$ whose components are, in that given order, the mass of the cell, the START promoting factor, a complex made of the so-called A-type cyclins, the mitosis promoting factor, a complex made of Btype cyclins and a specific ubiquitin pathway, which regulates the destruction of $B$-types cyclins.

There are three sets of equations: one governs the dynamics of $x$; the second linear set is made of the transport equations which determine the evolution of cells during the periods when they are subject to growth and mortality only. The third set of equations describes the transition from pre-START to post-START, on the one hand, and the division (or transitions post-START to pre-START of the next generation), on the other hand. In the absence of stochastic effects in the transitions, the passage through START occurs for some value of the cellcycle state $x$ which is entirely determined by the initial value. The same is true for the value at division. According to Val and Tyson (in preparation) START is a signal indicating that the cell is committed irrevocably to carry out DNA synthesis and engage into the sequence of events which ends with mitosis.

Looked at through several cycles, the cell-cycle state of a small cell (it is known that, at division, each cell of the budding yeast produces a large cell and a small one) is a piecewise continuous function with jumps at division times. Between two consecutive jumps, it satisfies the system of equations associated with the dynamics of $x$. It is reported by Val and Tyson (in preparation) that the cell-cycle state trajectories of all small cells approaches a similar cyclic attractor, and it is conjectured that there is a stable size distribution.

\section{Two Subcycle Cell-Cycle Model}

This model is based on the assumption that the cell cycle constitutes two loosely coupled subcycles. One is the DNA division cycle (DDC) or chromosome cycle, including G2 and M phases and a pre-S phase. The other subcycle is a cell-growth cycle (CGC) or cytoplasmic cycle, in which cellular mass is doubled. Both subcycles are necessary to produce new cells. DDC has a fairly constant duration, while CGC varies considerably in growth rate, as found experimentally. These subcycles are assumed to be mutually dissociated, being possible that their completion are not simultaneous. If subcycle CGC is finished before DDC is completed, it is possible that the new S-phase starts, generating a tetraploid cell. Sennerstam and Stromberg (1995a,b) present an explanation of this model and simulation results are also provided.

\section{CLASSICAL LINEAR MODELS OF STRUCTURED CELL POPULATION DYNAMICS}

We introduce in this section some ideas in the formulation of classical models of cell-population dynamics, following the survey of Arino (1995). This work provides a review of classical results together with a list of references. 
Structured models in cell-population dynamics were considered for the first time in the 1960s. The first paper on this subject seems to be by Bell and Anderson (1967). Work done during the 1970s turns mostly around non-linear models, but this situation changes in the beginning of the 1980s when linear models of population dynamics began to florish in various domains of applications, notably in cellpopulation dynamics. Most studies culminate in the proof of stable-type distribution property or asynchronous exponential growth (AEG). In a relatively very short period of time, many authors identified independently the main mathematical problems to be solved in this context and the main tools to be used (e.g. Pruss, 1981; Diekmann, 1982: Webb, 1983). A number of books, amongst which are Webb (1985), Metz and Diekmann (1986) and Lasota and Mackey (1985, 1994), illustrate the rapid development of the subject. Amongst the reasons for such a development, is the progress made earlier in the theory of positive operators. Interestingly, it appears that population dynamics played also its role in the theory of positive operators by giving a strong motivation for getting new results or improving the formulation of older ones. Good examples of this effect are the books by Nagel (1986) and Clément et al. (1987).

Roughly speaking, there are three approaches to the classical models in cell-population dynamics: cell-generation models, size-density models and time continuous daughter-cell models. In all cases, the main assumptions which can be made are:

(a) That each of the two daughters of a dividing cell gets the same share of the non-genetic material of the mother. This situation corresponds to equal division. The alternative situation is called unequal division.

(b) That a cell may or may not alternate between active periods, so-called proliferative during which it grows and divides, and dormant or quiescent, when it does not grow or divide.

(c) That the material of a cell grows exponentially fast or not. It does if the growth rate function is linear.

(d) That the lifelength of a cell is determined or stochastic.
For each of the above four alternative statements, one of them favours AEG, while in some instances when the other choice is taken, AEG does not hold.

\section{Cell Generation Models (Tyson and Hannsgen; Lasota and Mackey)}

In this model, the cell cycle is divided into two phases; phase A occupies part of the G1 phase. During this first phase, the cell grows until it reaches a size which for all cells is the same, normalized to the value 1 (unit of size). From this moment on, it stays in phase A during a time $T_{\mathrm{A}}$ which has a random distribution. It then enters phase $\mathrm{B}$. Phase $\mathrm{B}$ covers part of G1, the whole $S$ phase, G2 and goes to the end of mitosis. It lasts for a fixed period of time $T_{\mathrm{B}}$, the same for all cells (Smith and Martin, 1973).

Time $T_{\mathrm{A}}$ follows a Poisson law with parameter $p$ :

$$
\text { Prob }\left\{T_{\mathrm{A}}>t\right\}=\exp (-p t)
$$

so that $p^{-1}$ is the average length of phase $\mathrm{A}$, from the moment when cells reach size 1 . Size of cell is supposed to grow continually during both phases. Denoting $\tau$ the doubling size time, it is assumed that $T_{\mathrm{B}}<\tau$. Also, equal division is assumed, i.e. each daughter cell has exactly half the size of the mother.

From the above considerations, one can deduce that cells have a minimal size $\sigma$.

The model concentrates on describing the evolution of generations of cells. This implies that the time variable is the number of generations $(n \in \mathbf{N})$, not the chronological time. The initial time is the zeroth generation: all cells present at this initial stage are taken as the ancestor cells. To each number $n$, one associates a probability density function $\psi_{n}$ obtained by counting the cells produced by $n$ successive divisions, starting from ancestor cells, and calculating the proportion of those cells of each size.

The so-called dynamical process is the process by which cells pass from $n^{\text {th }}$ to $(n+1)^{\text {th }}$ generation. It is reflected in the densities by a transformation which gives $\psi_{n+1}$ in terms of $\psi_{n}$. This transformation is completely determined by the mechanism by which individual cells progress through the cycle that we explained before. The derivation is done in Tyson and Hannsgen (1986) and we refer to this for details. 
The above transformation is expressed in terms of a kernel $K(x, y)$, so that

$$
\psi_{n+1}(x)=\int_{\sigma}^{+\infty} K(x, y) \psi_{n}(y) \mathrm{d} y
$$

which determines a linear positive operator $P$ on $L^{1}(\sigma,+\infty)$ which leaves the subset of probability densities invariant. Such an operator is called a Markoy operator (Lasota and Mackey 1994).

The stable-size distribution problem can be formulated as follows:

To prove existence of a positive fixed point $\psi^{*}$ of operator $P$ (a steady-state distribution), and show that $\psi^{*}$ is globally asymptotically stable, that is, show that for each initial probability density $\psi_{0}, \psi_{n}$ tends to $\psi^{*}$, as $n$ goes to $+\infty$.

Conditions are stated in the above-mentioned book (Lasota and Mackey, 1994) in order to answer positively this problem.

\section{Size-Density Models}

The best account on size-density models is probably the book by Metz and Diekmann (1986). There is some connection with fluid dynamics in this approach, namely individuals are like particles of a fluid which flow into a region, which is the space of all the structure variables. In a single-type model (Diekmann et al., 1984), the state variable is a scalar density function $n(t, x)$, where the structure variable $x$ may be a vector or a scalar. A standard choice is the vector $(a, s)$ where $a$ represents the age and $s$ is a scalar associated with the measure of some other character.

This structure variable belongs to some fixed region $\Omega \subset \mathbf{R}^{N}$ ( $N$ is the dimension of the vector $x$ ) and we assume that the motion of particles in $\Omega$ is governed by an O.D.E.:

$$
\frac{\mathrm{d} x}{\mathrm{~d} t}=g(x(t))
$$

The model is obtained counting the number of individuals entering or leaving any region $M$ of $\Omega$ during a time interval $(t, t+\delta t)$, jointly with a standard application of the Stokes theorem, which enables us to express the evolution of the population as a transport equation:

$$
\frac{\partial n}{\partial t}+\frac{\partial}{\partial x}[n(t, x) g(x)]=B(t, x)-D(t, x) .
$$

$B$ and $D$ correspond respectively to source terms and sink terms. In order to describe them, we introduce three functions:

(a) The division rate $b(x)$ (per unit of time/per cell of size $x$ )

$$
\begin{aligned}
& \iint_{\left[x_{1}, x_{2}\right] \times\left[t_{1}, t_{2}\right]} b(x) n(t, x) \mathrm{d} x \mathrm{~d} t \\
& =\text { number of cells of size in }\left[x_{1}, x_{2}\right] \\
& \quad \text { which divide during the time } \\
& \quad \text { interval }\left[t_{1}, t_{2}\right] .
\end{aligned}
$$

(b) The mortality rate $\mu(x)$ (per unit of time/per cell of size $x$ ).

(c) The dispersion of cells at division amongst the two daughter cells (unequal division). It is defined in terms of a conditional density $f(x, y)$ which gives the distribution of the size of a daughter cell $(x)$, when the size of the mother is equal to $(y)$ :

$$
\begin{array}{rl}
\int_{x_{1}}^{x_{2}} & f(x, y) \mathrm{d} x \\
= & \text { probability for a daughter cell to have a } \\
& \text { size in the interval }\left[x_{1}, x_{2}\right] \text { knowing that } \\
& \text { the mother had size } y .
\end{array}
$$

Two obvious properties that such a distribution should verify are $f(x, y)=0$ if $x>y$ (the size of the daughter is less than that of the mother) and $f(x, y)=f(y-x, y)$ (the distribution is symmetric, due to the fact that as soon as the size of one of the two daughters is known, the other one is also known in terms of the size of the mother).

With these hypotheses, equation (1) takes the form

$$
\begin{aligned}
& \frac{\partial n}{\partial t}+\frac{\partial}{\partial x}[n(t, x) g(x)] \\
& =2 \int_{0}^{+\infty} f(x, y) b(y) n(t, y) \mathrm{d} y \\
& \quad-[b(x)+\mu(x)] n(t, x) .
\end{aligned}
$$


In case of equal division, the distribution $f$ reduces to a Dirac function

$$
f(x, y)=\delta\left(\frac{x-y}{2}\right) .
$$

In order to rigorously derive the corresponding model for equation (2), it is enough to write an approximation of $\delta$ by a true function and after passage to the corresponding limit we obtain the following equation

$$
\begin{aligned}
& \frac{\partial n}{\partial t}+\frac{\partial}{\partial x}[n(t, x) g(x)] \\
& =4 b(2 x) n(t, 2 x)-[b(x)+\mu(x)] n(t, x) .
\end{aligned}
$$

A natural assumption is that division occurs only in a certain size interval, so that $b(x)=0$ if $x<x_{0}$ or $x>x_{1}$ for some $0<x_{0}<x_{1}<+\infty$.

Moreover, $\left(x_{1} / 2\right)<x_{0}$, so that all cells have to grow for some time before they can divide. Cells may exceed size $x_{1}$ but cells of size larger than $x_{1}$ do not divide anymore. So they can be treated as if they had left the cycle. Assuming that division is equal and no cell divides below size $x_{0}$, implies that daughter cells have a minimum size $x_{0} / 2$.

As a consequence, we obtain a boundary condition on the solutions

$$
n\left(t, x_{0} / 2\right)=0
$$

A detailed study of system (3)-(4) was undertaken by Diekmann et al. (1984).

The stable-size distribution is a solution of the problem which has the form

$$
n(t, x)=\mathrm{e}^{\lambda t} \varphi(x) .
$$

Greiner and Nagel (1988) proved the following result

Assume that the function $g$ verifies the property $g(2 x) \leq 2 g(x)$, for all $x \in\left[x_{b} / 2, x_{1} / 2\right]$. Then, system (3)-(4) has a stable-size distribution (in other words, has exponential asymptotic growth (AEG)), if the function $g$ is such that $g(2 x)<2 g(x)$ for some $x \in] x_{0} / 2, x_{1} / 2[$.
The condition $g(2 x) \leq 2 g(x)$ is biologically meaningful. In fact, $g(2 x)$ can be interpreted as the total growth rate of a mother cell of size $2 x$, while $2 g(x)$ could be seen as the total growth rate of the two daughter cells if the division were to take place when the mother has size $2 x$. Whether the strict inequality may hold is still an undecided issue. When it holds, cell-size growth cannot be linear. On the other hand, it seems to be commonly admitted amongst biologists that cell size grows exponentially during the unlimited cell-growth phase. This explains attempts made recently in order to allow for linear growth rate and have at the same time AEG. The general model (2)-(4) in which $f$ is a density of probability other than the Dirac function was considered by Heijmans (1984), who proved that AEG holds even in the case when $g$ is such that $g(2 x)=2 g(x)$.

Whether unequal division is an important factor in producing cell variability is still a controversial issue amongst biologists. A mechanism was recently analyzed, by which AEG can hold even in the case of both linear growth rate and equal division. It assumes that the total population can be subdivided into two categories of cells: the proliferative cells (P), whose size grows according to a certain growth law, and which are subject to division and the quiescent cells (Q), which do not grow and do not divide. A model allowing for such a mechanism, motivated by the study of tumour cells was first derived by Gyllenberg and Webb (1987) and analyzed by Gyllenberg and Webb (1990, 1991). They prove that AEG holds under some assumptions. Different versions of this model are considered by Rossa (1991, 1995). In all of them, equal division is assumed.

\section{Time-Continuous Daughter-Cell Models}

We now review models describing the evolution of cells in one specific state: either, at the end of growth when they are mother cells, or, just after division, when they are daughter cells. A first model of this type was proposed by Kimmel et al. (1984) aiming mainly at showing the influence of unequal division 
on the dispersion of cell size through successive generations. Unequal division is modelled by a function $f(x, y)$ similar to the function considered above, which in addition satisfies a support property: There exists $0<d<1 / 2$ such that $f(x, y)>0$ if $d y<x<(1-d) y$.

The cell cycle is modeled by means of two functions

$$
T=\psi(x)
$$

the length (or duration) of the cell cycle of any cell entering the cycle with size $x$. It is assumed that $\psi$ is decreasing and

$$
0<\psi(+\infty) \leq \psi(x) \leq \psi(0) .
$$

The other function

$$
y=\Phi(x)
$$

gives the final size, that is, the mother size, as a function of the initial size. It is assumed that $\Phi$ is increasing, bounded and continuously differentiable with $\Phi(0)>1$.

The state variable of the model is $n(t, y)=$ density of daughter cells with respect to size and time.

Also important in the derivation of the model is $m(t, y)=$ density of mother cells with respect to size and time.

$n$ and $m$ are related to each other by two fundamental equations

$$
\begin{aligned}
& n(t, x)=2 \int_{0}^{+\infty} f(x, y) m(t, y) \mathrm{d} y \\
& m(t, y)=n\left(t-\psi \circ \Phi^{-1}(y), \Phi^{-1}(y)\right)\left(\Phi^{-1}\right)^{\prime}(y)(6)
\end{aligned}
$$

Equation (5) is straightforward and equation (6) is obtained by counting the number of mother cells

$$
\iint_{\left[t_{1}, t_{2}\right] \times\left[y_{1}, y_{2}\right]} m(t, y) \mathrm{d} t \mathrm{~d} y
$$

in terms of the daughter-cell density. We refer to Kimmel et al. (1984) and Arino and Kimmel (1987) for a detailed treatment of this computation.
Substituting the right-hand side of equation (6) for $m$ in equation (5), we obtain an equation involving $n$ only. After a change of variables in the integral, it takes the form

$$
n(t, x)=2 \int_{0}^{+\infty} f(x, \Phi(z)) n(t-\psi(z), z) \mathrm{d} z .
$$

The condition on the support of $f$ yields a support property for the solutions. Under the additional assumption

$$
d \Phi^{\prime}(0)>1
$$

one can show that, for any interval $] A_{1}, A_{2}[, 0<$ $A_{1}<A_{2}<+\infty$, with $A_{1}$ small enough and $A_{2}$ large enough, if we assume that daughter cells born before some time $t_{0}$ had size in $] A_{1}, A_{2}[$, then the same is true for all cells born after that time. Moreover, the size of daughter cells evolves in such a way that asymptotically it belongs to an interval $] a_{1}, a_{2}[$, where $a_{1}$ (or $a_{2}$ ) is the non-zero fixed point of $d \Phi$ (or $(1-d) \Phi)$.

Assuming that daughter-cell sizes are in some interval $] A_{1}, A_{2}[$, it was proved in Arino and Kimmel (1987) that AEG holds if the function

$$
\theta=\psi \circ \Phi^{-1}
$$

is such that

$$
\left.\left|\theta^{\prime}(y)\right| \geq \theta_{0}^{\prime}>0 \text { for all } y \in\right] A_{1}, A_{2}[.
$$

On the other hand, AEG does not hold if we assume that

$$
\left.\theta(y)=\theta_{0} \text { for all } y \in\right] A_{1}, A_{2}[
$$

The function $\theta(y)$ has a biological meaning, it gives the lifelength of a cell which at mitosis has size $y$.

The equal division version of equation (7) is

$$
n(t, x)=4\left(\Phi^{-1}\right)^{\prime}(2 x) n\left(t-\theta(2 x), \Phi^{-1}(2 x)\right) .
$$

Under the assumption that $\Phi^{-1}(2 x)$ has a positive fixed point $a^{*}$, one can see that AEG does not hold.

The model introduced here was mainly intended to reflect the impact of unequal division on AEG. It turns out from version (8) that, in this model, unequal division is in fact necessary to get AEG. 
A more general model which yields AEG even in the case of equal division, was introduced in Arino et al. (1991). In this model, stochastic lifelength is considered. Let us consider the case of unequal division, still modeled by the same function $f$ as above.

The cell-cycle duration is modeled by a conditional probability, expressed in terms of a density $\gamma(\cdot, x)$ :

$$
\begin{aligned}
& \int_{\left[\tau_{1}, \tau_{2}\right]} \gamma(\tau, x) \mathrm{d} \tau \\
& =\text { probability for a cell of initial size } x \text { to } \\
& \quad \text { have a cell cycle of length } \tau \in\left[\tau_{1}, \tau_{2}\right] .
\end{aligned}
$$

Moreover, $\gamma$ satisfies a technical support property. It is also assumed that the function $\Phi$ depends on $\tau$ and $x$ and $\Phi$ is also increasing with respect to $\tau$. Equation (7) is changed to

$n(t, x)=2 \iint_{\mathbf{R}^{+} \times \mathbf{R}^{+}} f(x, \Phi(\tau, y)) n(t-\tau, y) \mathrm{d} y \mathrm{~d} \tau$

It was proved in Arino et al. (1991) that, under some conditions stated on $f, \Phi$ and $\gamma$, AEG holds.

The equal division version of equation (9) is formulated by Arino (1995) and proves that AEG holds.

\section{Comparison Between the Three Approaches}

\section{Comparative remarks about the cell-growth law}

Kimmel et al. (1984) and subsequent work by Arino and Kimmel assume that cell growth is modeled in terms of a function $\Phi=\Phi(x)$ or $\Phi=\Phi(\tau, x)$. If we assume that cell growth is governed by a differential equation

$$
\frac{\mathrm{d} x}{\mathrm{~d} t}=g(x(t))
$$

and we denote $X(t, x)$ the flow associated to the equation, then $X(t, x)$ plays the same role as $\Phi(\tau, x)$ in Arino et al. (1991).

Conversely, it was proved by Arino and Kimmel (1993) that to a function $\Phi(\tau, x)$ it can be associated a non-autonomous differential equation

$$
\frac{\mathrm{d} x}{\mathrm{~d} t}=g(t, x(t))
$$

The flow $X(t, s, x)$ determined by the differential equation is related to $\Phi$ as follows:

$$
X(t, s, x)=\Phi\left(t, \Phi^{-1}(s, x)\right) .
$$

In particular, we have $X(t, 0, x)=\Phi(t, x)$.

So, we can conclude that the model with $\Phi=$ $\Phi(\tau, x)$ is in fact more general than the differential equation.

\section{$P D E$ versus integral equation}

Size-density models are expressed in terms of PDE (partial differential equation). In contrast, timecontinuous daughter-cell models lead to integral equations.

Arino and Kimmel (1993) prove that the integral equation (9) is equivalent to a first-order PDE involving the derivatives with respect to time, size and age, through a one-to-one correspondence between the solutions of (9) and the solutions of PDE. Such a comparison had be made previously by Webb (1987).

\section{Time-continuous versus discrete generation models}

The two approaches, the time-continuous approach and the cell-generation one, are completely different ways of cell counting. Strictly speaking, cellgeneration models are not evolution models: cells of a given generation are not in general contemporary to each other. Even when starting from a single cell, after a time large enough for asynchronicity to take on its effect, a large number of generations coexist on the one hand, while, on the other hand, cells of a same generation may be present at quite different times. Although the two approaches are so different, it is natural to think that they are somehow related to each other. Ideally, one should be able to describe cell generations in terms of a cell-population time map and conversely. It seems to be however that not all the models allow this. A basic requirement is that the model should be able to distinguish cells by their state in the cycle. The models described above do not feature these traits since the state variable $n(t, x)$ accounts for all cells 
which, at time $t$, have the same size $x$ independent on what part of the cell cycle they are in. On the other hand, time-continuous daughter models as the one described above allow a description in terms of generations. Such a description was done by Kimmel and Axelrod (1991), in a framework slightly! different from the one described above. We will now review the work by Kimmel and Axelrod (1991), introducing occasionally some modifications in their presentation in a way which copes more closely with assumptions and the analysis made by Arino and Kimmel (1987).

The model by Kimmel and Axelrod (1991) is described in terms of three functions:

(a) the function $\Phi$ which, as noted above, gives the size at division as an increasing function of the initial size.

(b) A function $G$, a cumulative distribution function, governing a random dispersion of the size at division with respect to the initial size. This means that in the Kimmel-Axelrod model, the sizd at division $y$ is expressed in terms of the size at birth $(x)$ by the following

$$
y=\Phi(x)+v
$$

where $v$ is a stochastic disturbance.

$$
\text { Prob }\{v \leq V\}=G(V) \text {. }
$$

No random dispersion corresponds to $G(V)=0$ for $V<0, G(V)=1$ for $V>0$.

(c) A function $H$, a cumulative distribution function, governing a random dispersion of the sizes of the daughter cells with respect to the size of the imother.

Let 4 s immediately compare these assumptions to those made previously. There is no equivalent to function $G$ : no dispersion is assumed with respect to the initial size. Function $H$, on the other hand, is strongly connected to the conditional density $f(x, y)$. In fact, the assumption made by Kimmel and Axelrod (1991) turns out to be a special case of that of Arino and Kimmel (1987).

Kimmel and Axelrod (1991) show unequal division to be modelled as follows: From a mother of size $y$, it is assumed that one of the daughter cells has the size $u y$, where $u$ is a random variable with values in $[0,1]$, the size of the other cell being, of course, $(1-u) y$. The law of $u$ is given by the function $H$ :

$$
\text { Prob }\{u \leq U\}=H(U) .
$$

Assuming this law, we can compute:

$$
\begin{aligned}
\operatorname{Prob}\left\{x_{1} \leq x \leq x_{2} \mid y=Y\right\} & =\operatorname{Prob}\left\{\frac{x_{1}}{Y} \leq u \leq \frac{x_{2}}{Y}\right\} \\
& =H\left(\frac{x_{2}}{Y}\right)-H\left(\frac{x_{1}}{Y}\right) .
\end{aligned}
$$

In terms of the function $f$, we have

$$
\text { Prob }\left\{x_{1} \leq x \leq x_{2} \mid y=Y\right\}=\int_{x_{1}}^{x_{2}} f(x, Y) \mathrm{d} x .
$$

This yields the equality

$$
\int_{x_{1}}^{x_{2}} f(x, Y) \mathrm{d} x=H\left(\frac{x_{2}}{Y}\right)-H\left(\frac{x_{1}}{Y}\right)
$$

which, in particular, entails that $H$ is absolutely continuous, and its density $h$ satisfies the following relationship

$$
f(x, y)=\frac{1}{y} h\left(\frac{x}{y}\right)
$$

So, the assumption made by Kimmel and Axelrod (1991) is a special case of the one made by Arino and Kimmel (1987). It corresponds to the case when the function $y f(x, y)$ is positively homogeneous of degree 0 in $(x, y)$.

An important difference between the two models is the absence in (Kimmel and Axelrod, 1991) of a function $\psi$ giving the duration of the cycle, in contrast to what was assumed previously (Arino and Kimmel, 1987). This absence is justified by the fact that the generation model does not account for the time spent by cells in their cycle. It indicates that that model carries less information than the timecontinuous daughter model and that one cannot, in general, derive the latter one in terms of the generation model.

Another difference between the two models is that Kimmel and Axelrod (1991) envisaged mortality and quiescence of daughter cells, while these factors were not considered by Arino and Kimmel (1987). 
It is assumed that of all the daughter cells, a fraction $p$ is going to grow and divide, a fraction $q$ will die while the remaining $r^{\text {th }}$ part becomes quiescent; $p q$ and $r$ being three constant numbers, with $p+q+r=$ 1. It is also assumed that, of all the quiescent cells, a fraction $s$ is going back into the proliferating state while the remaining $(1-s)^{\text {th }}$ part stays in quiescence.

Recurrence formulae for successive generations of proliferating and quiescent cells were obtained by Kimmel and Axelrod (1991) using a branching process approach. We will obtain the same result using a direct probability argument, in the restricted case when the quiescent cells do not return to the proliferating state, i.e. $s=0$. As a matter of fact, the case when quiescent cells are allowed to become proliferating seems to bear some difficulties. As long as it is quiescent, a cell keeps the same generation number. So, assuming a stochastic return to the proliferating state, one can have cells with a low generation number at arbitrarily large times. A full description of cells of any given generation will necessitate the computation of cells of that generation through all positive times and seems to be subject to some information on how long it takes to quiescent cells to become proliferating.

We start from an initial generation, the zeroth generation, whose distribution over the cell size is a function $D_{0}(\zeta)\left(D_{i}(\zeta)\right.$ is the distribution for the $i^{\text {th }}$ generation). The $D_{i}$ are the states at birth. We denote $M_{0}(\zeta)$ the initial distribution of cells at division, the mother cells $\left(M_{i}(\zeta)\right.$ is the distribution for the $i^{\text {th }}$ generation).

The word initial refers here to the first generation: the zeroth generation of mother cells follows the zeroth generation of daughter cells. In order to compute $M_{0}$ in terms of $D_{0}$, we first give an expression of the size at division as a function of the size at birth. It is easy to see that

$$
\begin{aligned}
\text { Prob }\left\{y \in\left[y_{1}, y_{2}\right] \mid x=X\right\} \\
=\operatorname{Prob}\left\{y_{1} \leq \Phi(X)+v \leq y_{2}\right\} \\
=G\left(y_{2}-\Phi(X)\right)-G\left(y_{1}-\Phi(Y)\right) .
\end{aligned}
$$

It corresponds to the probability density $\mathrm{d} G(Y-\boldsymbol{\Phi}$ $(X))$ or $g(Y-\Phi(X))$ d $Y$ if $G$ is absolutely continuous.
Counting the mother cells with mass between $y_{1}$ and $y_{2}$ in terms of the daughter-cell density yields

$$
\begin{aligned}
& \int_{y_{1}}^{y_{2}} M_{0}(y) \mathrm{d} y \\
& \quad=\int_{0}^{+\infty}\left(\int_{y_{1}}^{y_{2}} \mathrm{~d} G(y-\Phi(x)) D_{0}(x)\right) \mathrm{d} x \\
& =\int_{0}^{+\infty}\left(\int_{y_{1}}^{y_{2}} g(y-\Phi(x)) D_{0}(x) \mathrm{d} y\right) \mathrm{d} x .
\end{aligned}
$$

So, assuming that $G$ is absolutely continuous, we arrive at

$$
M_{0}(Y)=\int_{0}^{+\infty} g(Y-\Phi(x)) D_{0}(x) \mathrm{d} x .
$$

If there is no random dispersion of the sizes, i.e. $G(v)=0$ if $v<0, G(v)=1$ if $v \geq 0$, the above formula reduces to

$$
M_{0}(Y)=\left(\Phi^{-1}\right)^{\prime}(Y) D_{0}\left(\Phi^{-1}(Y)\right) .
$$

After division, mother cells of the zeroth generation are followed by daughter cells of the first generation, surviving with probability $p$. This leads to

$$
D_{1}(x)=2 p \int_{0}^{+\infty} f(x, y) M_{0}(y) \mathrm{d} y .
$$

Substituting for $M_{0}$ its expression in terms of $D_{0}$, we obtain a relationship between two consecutive generations of cells which determines a discrete dynamical system. The transition from the $i^{\text {th }}$ to the $(i+1)^{\text {th }}$ generation is given by the following

$$
\begin{gathered}
D_{i+1}(x)=2 p \int_{0}^{+\infty} \int_{0}^{+\infty} f(x, y) \\
\times g(y-\Phi(z)) D_{i}(z) \mathrm{d} z \mathrm{~d} y .
\end{gathered}
$$

In the case considered by Kimmel and Axelrod (1991), it yields

$$
\begin{aligned}
& D_{i+1}(x)=2 p \int_{0}^{+\infty} \int_{0}^{+\infty} \frac{1}{y} h\left(\frac{x}{y}\right) \\
& \times g(y-\Phi(z)) D_{i}(z) \mathrm{d} z \mathrm{~d} y .
\end{aligned}
$$

This is the same as formula (A.10) of Kimmel and Axelrod (1991). 


\section{NON-LINEAR MODELS}

We now present some non-linear modified versions of time-continuous daughter-cell models developed by Arino and Kimmel $(1989,1991)$. These models include non-linear dependence of the fraction of cells undergoing effective division on the total number of cells in the cycle.

\section{Non-Linear Functional-Integral Equation of Cell Kinetics with Unequal Division}

We keep notations and hypotheses of the section on 'Time-continuous daughter-cell models' and we add the following assumptions:

(a) After division, a proportion $\sigma$ of daughter cells return to the cycle, while the rest (fraction $1-\sigma$ ) choose another development path, for example, they differentiate. $\sigma$ is a function of the total number of the cycling cells, $\sigma=\sigma(N)$. The peak value of $\sigma$ is 1 .

(b) There exists a probability of a defective division. The probability of this fact is $1-\lambda$. Thus, $\lambda \in[0,1]$ is the probability of correct division.

Based on these assumptions, equation (5) becomes

$$
n(t, y)=2 \lambda \sigma(N(t)) \int_{0}^{+\infty} f(y, x) m(t, x) \mathrm{d} x
$$

and then, equation (7) has to be changed to

$$
\begin{aligned}
& n(t, y)=2 \lambda \sigma(N(t)) \int_{0}^{+\infty} f(y, \Phi(z)) \\
& \times n(t-\psi(z), z) \mathrm{d} z
\end{aligned}
$$

Notice that the number of cells of birthmass $x$ present in the cycle at time $t$ is equal to the number of cells of that mass which entered the cycle in the time interval from $t-\psi(x)$ to $t$. Integrating over $x$ yields

$$
N(t)=\int_{0}^{+\infty} \int_{t-\psi(x)}^{t} n(s, x) \mathrm{d} s \mathrm{~d} x .
$$

Equations (10) and (11) define the model completely and equation

$$
n(t, y)=\int_{a_{1}}^{a_{2}} f(y, \Phi(z)) n(t-\psi(z), z) \mathrm{d} z
$$

can be considered as a linear version of equation (11) describing the situation where each division results in one proliferating cell (on average), i.e. during zero population growth.

From equations (10) and (11) it is obtained, using the fact that $\int_{0}^{+\infty} f(y, x) \mathrm{d} y=1$, that

$$
\begin{aligned}
& \dot{N}(t)=[2 \lambda \sigma(N(t))-1] \\
& \quad \times \int_{\left(A_{1}, A_{2}\right)} n(t-\psi(z), z) \mathrm{d} z
\end{aligned}
$$

and then either $2 \lambda \sigma(N(+\infty))=1$ or $N(+\infty)=0$, where $N(+\infty)=\lim _{t \rightarrow+\infty} N(t)$. It can be shown that $N(+\infty)$ is completely determined in terms of combinations of parameters $\lambda$ and $N(0)$.

This model is analyzed in detail by Arino and Kimmel (1989) using the operator semigroup theory combined with a classical differential-equation approach. The non-negative solutions either tend to a stable steady state or to zero. It is not difficult to realize that results of the same completeness can be obtained for any regulation feedback function $\sigma$, provided it satisfies some technical hypotheses. The only difference is in the number and configuration of the equilibria. However, all of them have the form $N(+\infty) v_{0}(x)$.

The reason for this simplicity is that the non-linear aspects of the asymptotic behavior of the system (10) and (11) are reduced to the analysis of an ODE (13) for $N(t)$. The functional aspect of the asymptotic analysis is completely contained in the limit equation (12) which is nearly identical to the equation of unrestrained growth considered by Arino and Kimmel (1987). (It may be noticed, however, that the analysis of existence of solutions is more difficult for the non-linear problem.)

A modification of this model is proposed by Arino and Mortabit (1991) in which it is assumed that the non-linear dependence of the fraction of cells reentering proliferation is on the total number of cells that were in the cycle at time $t-1$. 
Equation (10) can be substituted by

$$
\begin{aligned}
& n(t, x)=2 \lambda \sigma(N(t-1)) \int_{0}^{+\infty} f(x, \Phi(z)) \\
& \times n(t-\psi(z), z) \mathrm{d} z
\end{aligned}
$$

and the total population satisfies a retarded differential equation

$$
\dot{N}(t)=[2 \lambda \sigma(N(t-1))-1] \int_{a_{1}}^{a_{2}} n(t-\psi(z), z) \mathrm{d} z
$$

This model provides an example where slow oscillations are forced into existence by the introduction of a delay term.

A very general model of cell-population dynamics, which includes the classical random transition, size control and inherited property models as special cases, is derived and analyzed by Webb (1987). Among specific applications of this model, the author includes the model by Kimmel et al. (1984) which serves as a basis of nonlinear model (10)-(11). It is interesting to remark that this model fits into the general approach only when the distribution of cell-generation time, which is one of the functions defining Webb's model, is replaced by Dirac's delta function. However, the study within the framework of Webb's paper seems to be difficult.

With respect to the concept of mitotic regulation, model (10)-(11) is analogous to Mackey's (1978) description of the proliferation of the stem cells of the hemopoietic system. The behavior of the population predicted by both models is, however, completely different: Mackey's model is capable of producing periodic oscillations while $(10)-(11)$ predict a stable regime or extinction of the population.

\section{Selective Regulation of Cell-Population Growth}

A more sophisticated and difficult nonlinear model was introduced by Arino and Kimmel (1991). The cells leave proliferation with probability increasing with either the total cell mass or the amount of a cellular constituent, RNA, selected protein, etc. This process is called selective regulation of cell population growth, and is in a qualitative agreement with observations in many biological cell systems.

The following assumption distinguishes the model and replace hypothesis (a) on page 45 .

(a') After division, a proportion of daughter cells return to the cycle, while the rest choose another development path, for example, they differentiate. Cells staying in the cycle are, with the highest probability, those with the lower $x$.

The probability of entering $\mathrm{Gl}$ is equal to $H(\Pi-x)$, where $H$ is a sigmoidal smooth approximation of the Heaviside function, increasing, with $H(-\infty)=0 . H(+\infty)=1 . \Pi($.$) is a$ decreasing function of the total number of cycling cells, i.e. $\Pi=\Pi(N)$.

Finally, the fraction entering $\mathrm{G} 1$, among cells with mass $x$ is $H(\Pi(N(t))-x)$.

Equation (10) has to be changed to

$$
\begin{aligned}
& n(t, y)=2 \lambda H(\Pi(N(t))-y) \\
& \quad \int_{0}^{+\infty} f(y, \Phi(z)) n(t-\psi(z), z) \mathrm{d} z
\end{aligned}
$$

and the model is completely determined by equations (11)-(14).

From the mathematical viewpoint, the stability analysis carried out by Arino and Kimmel (1991) has required novel tools of analysis of the spectral properties of semigroup of operators.

The solutions of the model equations generate a semigroup of non-linear positive operators. A trivial equilibrium exists for all $\lambda \in[0,1]$ and a non-trivial steady-state branches from it at $\lambda=$ $1 / 2$ and continues to exist for $\lambda \in[1 / 2,1]$. For $\lambda \in[0,1 / 2]$, the trivial solution attracts all the nonnegative solutions.

The study of the stability of the non-trivial equilibrium has motivated the elaboration of an original abstract result on the spectrum of a difference of two operators with certain non-negativity properties. In intuitive terms, it is shown that (under appropriate technical conditions), if $P$ is a linear operator with a simple eigenvalue 1 and with the rest of its spectrum constrained to a disc with radius $<1$, and if $R$ is an operator with certain positivity properties 
and not too large norm, then perturbing $P$ by $-R$ transforms the spectrum of $P$ in such a way that its spectral radius is $<1$.

From biological viewpoint, the so called selective regulation provides a tool for joint consideration of cell-population growth and maturation of individual cells.

The oldest concept of homeostatic regulation of the number of cells in a population seems to be that of mithtic autoregulation (Wheldon, 1988). Suppose that each cell synthesizes at a constant rate a molecular species which acts as an inhibitor of proliferation in the cells concerned. Suppose also that such molecules are both exported into the environment by the cell and reabsorbed from it. The intracellular density concentration depends on the density of inhibitor-synthesizing cells in the neighbourhood, which provides a negative-feedback mechanism. The inhibitory molecules may be thought of as maintaining the cell in a proliferative resting state. Many models exploited this concept (Mackey, 1978; Arino and Kimmel, 1986), which has the disadvantage of not taking into account any interdependence between regulation of cell number and maturation of individual cells.

It seems, however, probable that the maturation process is directly coupled with population regulation. Let us suppose that maturation means that the cell acquires a sufficient amount of a biochemical species. Cells which have enough of this substance leave proliferation. The regulatory mechanism may act in the following way: If the number of proliferating cells decreases, the threshold level of the substance required to leave proliferation is increased. Conversely, if the number of cells increases, the threshold level of the substance is decreased. These are the essential features of the model described before.

The basic question is whether a regulation feedback of this type could stabilize the number of proliferating stem cells and the distribution of the biochemical species in the population, under the influence of external perturbations. In the language of mathematics, this is the question of stability of equilibria. Arino and Kimmel (1991) answer this question in the affirmative, in a local sense. The existence of a stable equilibrium depends on a parameter $\lambda$ equal to the fraction of successful divisions. If this parameter is $<1 / 2$, the stem-cell population becomes extinct.

A common feature of many non-linear models is that the non-linearity is obtained by introducing dependence of some of the coefficients upon the total population. This frequently allows determining a sort of decoupled problem in terms of the total population and another variable corresponding to a structure-dependent density. First, the evolution of the total population can be investigated, frequently on its own. If a limit behavior of the total population can be derived from that study, then it frequently derives the behavior of the density. Thus, global behavior of the model can be obtained.

Arino and Kimmel (1989) show that the total population satisfies an ODE. In other approaches, a retarded equation has to be considered (Arino and Mortabit, 1991).

A recent example of a model with non-linearity depending upon the total population has been found (Mackey and Rudnicki, 1994). We provide a brief description of the model. The cell cycle is divided into two parts: a resting phase G0 during which cells are aging and growing in size (more precisely, here, in maturity, a rather abstract unspecified character associated with a variable with values in an interval $\left.\left[0, m_{F}\right], m_{F}<+\infty\right)$. and a proliferating phase during which replication of DNA and mitosis take place. Right after division, cells go into the resting phase where they can stay from 0 to $+\infty$, depending upon the total population of resting cells. The flux of resting population $(n)$ inside the proliferating compartment $(p)$ is given by

$$
p(t, m, 0)=\beta(\bar{N}(t), m) N(t, m)
$$

where $\bar{N}(t)$ is the total number of resting cells at all maturation levels, while $N(t, m)$ is the density of such cells with respect to the maturation variable.

Interestingly, the original model in $(n, p)$ can be somehow integrated to yield a system of equations in $N$ and $P$ (the density of proliferating cells with respect to the maturation variable). That system is a 
non-local PDE, the non-local terms being deviations both in time and the maturation variable.

Under further restriction on the coefficients, a delay-differential equation, is obtained for $\bar{N}$ by which it is possible to determine the behavior of $\bar{N}$ first, then that of $N$ and finally that of $n$ and $p$.

\section{CELL-CYCLE KINETICS WITH SUPRAMITOTIC CONTROL}

This section is intended to present a model which incorporates a two subcycles description of the cell cycle, and which is developed by Kimmel and Arino (1991). The model is based on the so-called supramitotic regulation, i.e. decisions controlling growth of the cell are made not at the beginning of the cell-division cycle but a previous point and their impact is extended to the next decision point, which is located in the next division cycle. The period from one decision point to the next is called the 'growth control cycle'. In this model, the new growth-control cycle, is entered when the cell attains a threshold size. The threshold is, in general, a random variable, so the model allows for imprecise control.

Another feature of the model is the presence of two types of cells. At the beginning of each growth-control cycle, cells may switch from one type to the other with given transition probabilities. This assumption is based on a theory of cell-cycle regulation for PPC 3 cells formulated by Sennerstam and Stromberg (1988). The main ideas are that cells may switch from a fast to a slow cell-cycle variant and back and that the decision is made in the preceding cell division cycle (supramitotic character of the regulation).

To derive the model, consider cells of type 1 (smaller) and of type 2 (larger) which may switch from type $i$ to type $j$ with a probability $p_{i j}$ at a sizecontrol point between cell divisions, for example on the G1/S phase boundary. Then they proceed to division, producing progeny of identical type. Therefore it is necessary to consider four variants of celldivision cycle $(1,1),(1,2),(2,1),(2,2)$, where $(i, j)$ denotes cells born as type $i$ that switched to type $j$.
It is assumed that the growth rate $r$ is constant throughout the cell cycle and identical for both cell types. Daughter cells entering Gl at size $y$ grow to a threshold size $w_{i}$, which is a random variable with distribution density $h_{i}$ depending on cell type $i$. The support of $h_{i}$ is a closed interval $\left[w_{i 1}, w_{i 2}\right]$ the ends of which are the minimum and maximum threshold values, respectively. Then the cells begin DNA synthesis, that is, they enter the S-phase. Parameters of the model will be chosen in a way that excludes the possibility of a daughter cell being equal to, or larger than, the minimum threshold for DNA synthesis. In other words, it is guaranteed that the $\mathrm{G} 1$ phase is longer than 0 .

After leaving G1, cells progress through phases S, G2 and M towards division. Total duration of these phases is assumed to be equal to $\tau$. During this time cells are still growing at rate $r$. The division is unequal, modelled by a distribution density $f(x, y)$ as described above.

The dynamics of the model is described in terms of distribution densities of cell flow rates through various points of the cell cycle. First, $n_{i j}(t, y)$ is the density of flow rate of the age 0 daughter cells into G1 phase, for the type $i$ cells that will switch to type $j$ at the G1/S boundary. The interpretation is that $n_{i j}(t, y) \mathrm{d} t \mathrm{~d} y$ is equal to the number of these cells with sizes in the interval $(y, y+\mathrm{d} y)$ that entered $\mathrm{G} 1$ in the time interval $(t, t+\mathrm{d} t)$.

Analogously, $m_{i j}(t, x)$ is the density of flow rate of mother cells through division. These are cells that started as type $i$ daughters and now are type $j$. Finally $q_{i j}(t, x)$ is the flow-rate density of daughter cells descending from mothers described above, before they are assigned to the G1 phase of any of the four cell-division cycle types.

The relationship between $n_{i j}$ and $q_{i j}$ is described by the system of equations

$n_{i j}(t, y)=p_{i j}\left[q_{1 i}(t, y)+q_{2 i}(t, y)\right], i=1,2$.

The principle of unequal division implies that the relationship between $q_{i, j}$ and $m_{i j}$ is

$$
q_{i j}(t, y)=2 \int_{0}^{+\infty} f(y, x) m_{i j}(t, x) \mathrm{d} x, i=1,2 .
$$


Finally, the distribution $m_{i j}$ of the flow rate of mother cells can be found from a balance argument which yields

$$
\begin{aligned}
& m_{i j}(t, x)=r h_{i}(x-\tau r) \\
& \quad \int_{0}^{w_{1 i}} n_{i j}(t-(\tau+\sigma), x-r(\tau+\sigma)) \mathrm{d} \sigma
\end{aligned}
$$

Combining equations (15), (16) and (17), we obtain

$$
\begin{aligned}
& n(t, y)=2 r \int_{0}^{+\infty} f(y, x) H(x-r \tau) \\
& \int n[t-(\tau+\sigma), x-r(\tau+\sigma)] \mathrm{d} \sigma \mathrm{d} x
\end{aligned}
$$

where we have used the matrix-vector notation

$$
n(t, y) \neq\left(n_{11}(t, y), n_{12}(t, y), n_{21}(t, y), n_{22}(t, y)\right)^{T}
$$

$H(w)$

$$
=\left[\begin{array}{cccc}
p_{11} h_{1}(w) & 0 & p_{11} h_{2}(w) & 0 \\
p_{12} h_{1}(w) & 0 & p_{12} h_{2}(w) & 0 \\
0 & p_{21} h_{1}(w) & 0 & p_{21} h_{2}(w) \\
0 & p_{22} h_{1}(w) & 0 & p_{22} h_{2}(w)
\end{array}\right] .
$$

The expression for the total number $N_{i j}(t)$ of the ( $i j)$-type cells present at time $t$ is derived in the following way. The density of cell flux into G1 including cells born with size $\zeta$ at time $\rho$ that will enter $\mathrm{S}$ after reaching threshold size $w$, is equal to $h_{i}(w) n_{i j}(\rho, \zeta)$. Population at time $t$ includes cells born before $t$ but not earlier than one cell-cycle duration before $t$, i.e. after $t-[\tau-(\omega-\zeta) / r]$. Eventually

$$
\begin{aligned}
& N_{i j}(t)=\int_{0}^{w_{1 i}} \int_{1 i}^{+\infty} \int_{t-[\tau-(w-\zeta) / r]}^{t} h_{i}(w) \\
& \times n_{i j}(\rho, \zeta) \mathrm{d} \rho \mathrm{d} w \mathrm{~d} \zeta
\end{aligned}
$$

where the upper bound $w_{1 i}$ reflects the fact that only cells with size less than the minimum threshold size are allowed into $\mathrm{G} 1$, by hypothesis.

This model is of independent mathematical interest. The analysis of asymptotic properties is accomplished by defining and investigating an abstract semigroup of positive linear operators in an appropriate state space. In brief, it is proved that the semigroup is eventually compact, that its spectrum has a dominating eigenvalue determined as a solution of a characteristic equation and that the asymptotic behavior of the semigroup is determined by the dominating eigenvalue. This allows conclusion of AEG for this model.

Kimmel and Arino (1991) make a comparison of the model with experimental data. Basic pedigree statistics, $\beta$ curves and generation time correlations are obtained from measurements available for the embryonic cells. The main biological conclusion which is consistent with these results is that switching from one cell type to the other is a necessary but relatively infrequent event.

\section{Remark}

The mechanism described by Kimmel and Arino (1991) for the switch is quite rudimentary. It is assumed that with a fixed probability, cells of a given type may either keep that type or switch to the other. This has the following consequence, with the notations used by Kimmel and Arino (1991).

$$
n_{12}(t, y)=\frac{p_{12}}{p_{11}} n_{11}(t, y), n_{21}(t, y)=\frac{p_{21}}{p_{22}} n_{22}(t, y)
$$

for $t$ large enough. Thus, the dynamics can be reduced to the dynamics of cells not changing type. This ceases to be true if it is assumed that the $p_{i j}$ depend on the size. In that case, the analysis made by Kimmel and Arino (1991) remains valid.

\section{References}

Arino, O. (1995) "A survey of structured cell population dynamics," Acta Biotheor., 43, 3-25.

Arino, O. and Kimmel, M. (1986) "Asymptotic analysis of models of cell production systems," Math. Modeling 7, 1269-1300. Arino, O. and Kimmel, M. (1987) "Asymptotic analysis of a cell cycle model based on unequal division," SIAM J. Appl. Math., 47, $128-145$

Arino, O. and Kimmel, M. (1989) "Asymptotic behavior of nonlinear functional-integral equation of cell kinetics with unequal division," J. Math. Biol., 27, 341-354.

Arino, O. and Kimmel, M. (1991) "Asymptotic behavior of nonlinear semigroup describing a model of selective cell growth regulation," J. Math. Biol., 29, 289-314.

Arino, O. and Mortabit, A. (1991) "Slow oscillations in a mode of cell population dynamics," Math. Pop. Dynamics. Lecture Notes Pure Appl. Math., 131, 13-27. Marcel Dekker, New York 
Arino, O. and Kimmel, M. (1993) "Comparison of approaches to modelling of cell population dynamics. SIAM J. Appl. Math., 53, $1480-1504$.

Arino, O. Kimmel, M. and Zerner, M. (1991) “Analysis of a cell population model with unequal division and random transition," Arino et al., eds. Proc. 2nd Int. Conf. Math. Pop. Dynamics. Marcel Dekker, New York.

Bell, G. I. and Anderson, E. C. (1967) "Cell growth and division. I. A mathematical model with applications to cell volume distribution in mammalian suspension cultures," Biophys. J., 7, 329-351.

Clément, Ph., Heijmans, H. J. A. M., Angenent, S., Van Duijn, C. J. and De Pagter, B. (1987) One-Parameter Semigroups. North-Holland, Amsterdam.

Diekmann, O. (1982) Lecture Notes Biomath. 54, 90-96. Springer-Verlag, Berlin.

Diekmann, O., Heijmans, H. J. A. M. and Thieme, H. R. (1984) "On the stability of the cell size distribution," J. Math. Biol., 19, 227-248.

Fantes, P. (1977) "Control of cell size and cycle time in Schizosaccharomyces pombe," J. Cell Sci., 24, 51-67.

Forsburg, S. L. and Nurse, P. (1991) "Cell cycle regulation in the yeasts Saccharomyces cerevisiae and Schizosaccharomyces Pombe," Ann. Rev. Cell Biol., 7, 227-256.

Greiner, G. and Nagel, R. (1988) "Growth of cell population via one-parameter semigroups of positive operators," Math. Appl. Sci., 79-105.

Gyllenberg, M. and Webb, G. (1987) "Age-Size structure in populations with quiescence," Math. Biosci., 86, 67-95.

Gyllenberg, M. and Webb, G. (1990) "A nonlinear structured population model of tumor growth with quiescence," J. Math. Biol., 28, 671-694.

Gyllenberg, M. and Webb, G. (1991) "Quiescence in structured population dynamics. Lecture Notes Pure Appl. Math., 131, 45-62. Marcel Dekker, New York.

Harvey, E. B. (1940) "A comparison of the development of nucleate and non-nucleate eggs of Arbacia punctulata," Biol. Bull., 79, 166-187.

Heijmans, H. J. A. M. (1984) "On the stable size distribution of populations reproducing by fission into two unequal parts," Math. Biosci., 72, 19-50.

Hyver, C. and Le Guyader, H. (1990) "MPF and cycling modelling of the cell cycle minimum oscillator," Biosystems, 24, 85-90.

John, P. C. L. (1981) The Cell Cycle, Cambridge University Press, London.

Kimmel, M. and Arino, O. (1991) "Cell cycle kinetics with supramitotic control, two cell types and unequal division: A model of transformed embryonic cells," Math. Biosci., 105, 47-79.

Kimmel, M. and Axelrod, D. (1991) "Unequal cell division, growth regulation and colony size of mammalian cells: A mathematical model and analysis of experimental data," $J$. Theor. Biol., 153, 157-162.

Kimmel, M., Darzynkiewicz, Z., Arino, O. and Traganos, F. (1984) "Analysis of a cell cycle model based on unequal division of metabolic constituents to daughter cells during cytokinesis," J. Theor. Biol., 110, 637-664.

Lasota, A. and Mackey, M. C. (1984) "Globally asymptotic properties of proliferating cell populations," J. Math. Biol., 19, 43-62.

Lasota, A. and Mackey, M. C. (1985) Probabilistic Properties of Deterministic Systems. Cambridge University Press, London.

Lasota, A. and Mackey, M. C. (1994) "Chaos. fractals and noise. Stochastic aspects of dynamics," Appl. Math. Sci., 97. Springer-Verlag, New York.
Mackey, M. C. (1978) "A unified hypothesis for the origin of aplastic anemia and periodic haematopoiesis," Blood, 51, 941-956.

Mackey, M. C. and Rudnicki, R. (1994) "Global stability in a delayed partial differential equation describing cellular replication," J. Math. Biol., 33, 89-109.

Metz, J. A. J. and Diekmann, O. (eds.) (1986) "Dynamics of physiologically structured populations," Lecture Notes Biomath., 86. Springer-Verlag, New York.

Mitchinson, J. M. (1971) The Biology of the Cell Cycle. Cambridge University Press, London.

Nagel, R. (ed.) (1986) "One-parameter semigroups of positive operators," Lecture Notes Math., 1184. Springer-Verlag, New York.

Norel, R. and Agur, Z. (1991) "A model for the adjustment of the mitotic clock by cyclin and MPF levels," Science, 251, 1076-1078.

Novak, B. and Tyson, J. J. (1995) "Mathematical modelling of the cell division cycle," Mathematical Population Dynamics: Analysis of Heterogeneity. Arino et al., eds. Wuerz, Canada.

Nurse, P. and Bisset, Y. (1981) "Gene required in G1 for commitment to cell cycle and control of mitosis in fission yeast," Nature 292, 558-560.

Obeyesekere, M., Tucker, S. L. and Zimmerman, S. O. (1992) "Mathematical models for the cellular concentrations of cyclin and MPF," Biochem. Biophys. Res. Comm., 184, 782-789.

Obeyesekere, M., Tucker, S. L. and Zimmerman, S. O. (1994) "A model for regulation of the cell cycle incorporating cyclin A, cyclin B and their complexes," Cell Proliferation, 27, $105-113$.

Obeyesekere, M., Tucker, S. L. and Zimmerman, S. O. (1995) "Mathematical models for regulation of the cell cycle via the concentrations of cellular proteins," Mathematical Population Dynamics: Analysis of Heterogeneity. Arino et ål., eds. Wuerz, Canada.

Pruss, J. (1981) "Equilibrium solutions of age-specific population dynamics of several species," J. Math. Biol., 11, 65-84.

Rossa, B. (1991) "Asynchronous Exponential Growth of Linear Co-Semigroups and a New Tumor Cell Population Model," $\mathrm{Ph} . \mathrm{D}$. Thesis, Vanderbilt University, USA.

Rossa, B. (1995) "Asynchronous exponential growth in a size structured cell population with quiescent compartment," Mathematical Population Dynamics: Analysis of Heterogeneity, 2, Arino et al., eds. Wuerz, Canada.

Sennerstam, R. and Stromberg, J. O. (1988) "Evidence for an intraconal random shift between two types of cell cycle times in an embryonal carcinoma cell line," J. Theor. Biol., 131, $151-162$.

Sennerstam, R. and Stromberg, J. O. (1995a) "Cell cycle progression: computer simulation of uncoupled subcycles of DNA replication and cell growth," J. Theor. Biol., 175, 177-189.

Sennerstam, R. and Stromberg, J. O. (1995b) "Contradictory conclusions from subcompartments of G1 phase are resolved by the two-subcycle cell cycle model," Mathematical Population Dynamics: Analysis of Heterogeneity. Arino et al., eds. Wuerz, Canada.

Smith, J. A. and Martin, L. (1973) "Do cells cycle?," Proc. Natl. Acad. Sci. USA, 70, 1263-1267.

Thron, C. D. (1991) "Mathematical analysis of a model of the mitotic clock," Science, 254, 122-123.

Thron, C. D. (in preparation) Modeling a bistable trigger of mitosis. Proc. 4th Conf. Math. Pop. Dynamics., Houston, 1995.

Tyson, J. J. (1991) "Modelling the cell division cycle: $c d c 2$ and cyclin interactions," Proc. Natl. Acad. Sci. USA, 88, $7328-7332$. 
Tyson, J. and Hannsgen, K. (1986) "Cell growth and division: a deterministic, probabilistic model of the cell cycle," J. Math. Biol., 23, 231-246.

$\mathrm{Val}, \mathrm{J}$. and Tyson, J. (in preparation) "A purely deterministic model for the population dynamics of budding yeast," Proc. 4th Conf. Math. Pop. Dynamics, Houston, 1995.

Webb, G. F. (1983) "A semigroup proof of the Sharpe-Lotka theorem,." Lecture Notes Math., 1076, 254-268. SpringerVerlag, Berlin.
Webb, G. F. (1985) "Theory of nonlinear age-dependent population dynamics. Pure Appl. Math., Series 89. Marcel Dekker, New York

Webb, G. F. (1987) "Random transitions, size control and inheritance in cell population dynamics," Math. Biosci., 85, $71-91$.

Wheldon, T. E. (1988) Mathematical Models in Cancer Research. Adam Hilger, Philadelphia. 


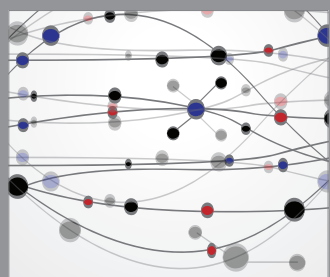

The Scientific World Journal
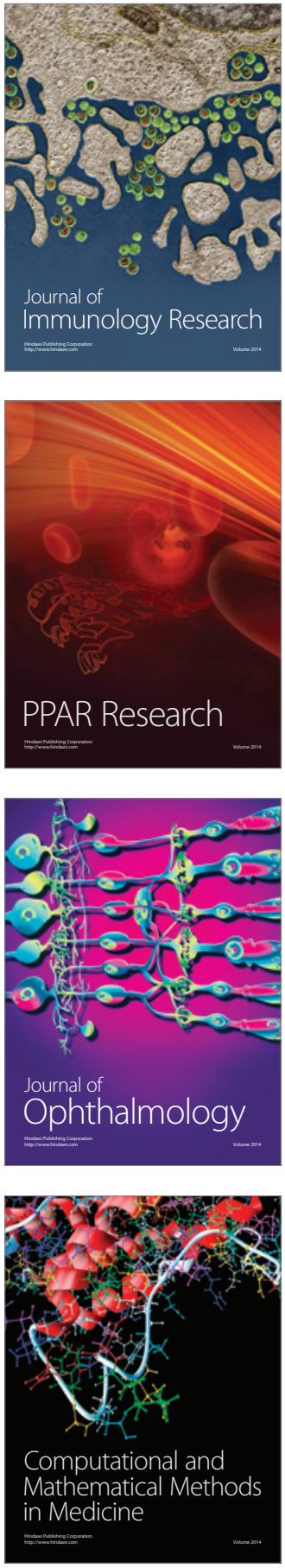

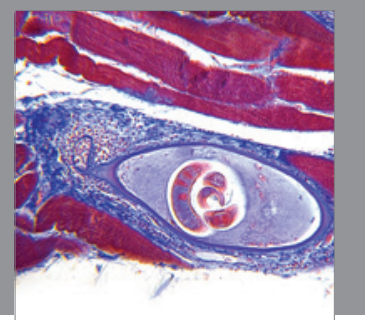

Gastroenterology

Research and Practice
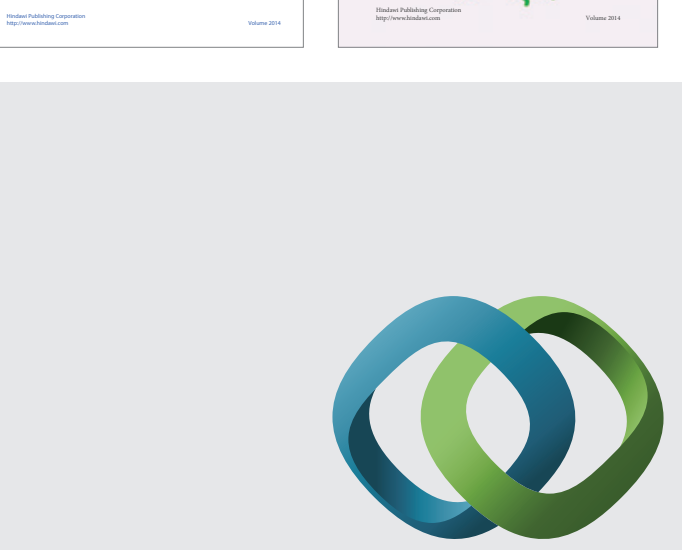

\section{Hindawi}

Submit your manuscripts at

http://www.hindawi.com
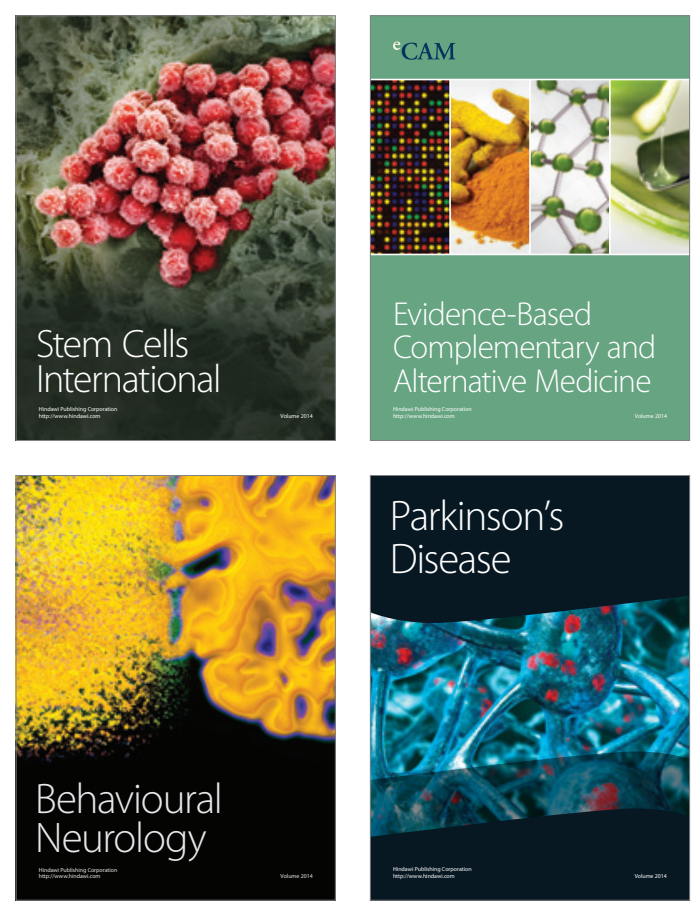

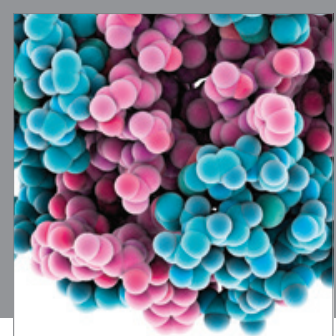

Journal of
Diabetes Research

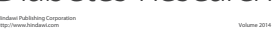

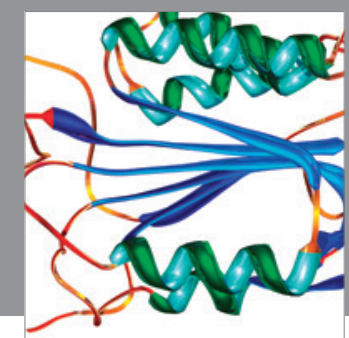

Disease Markers
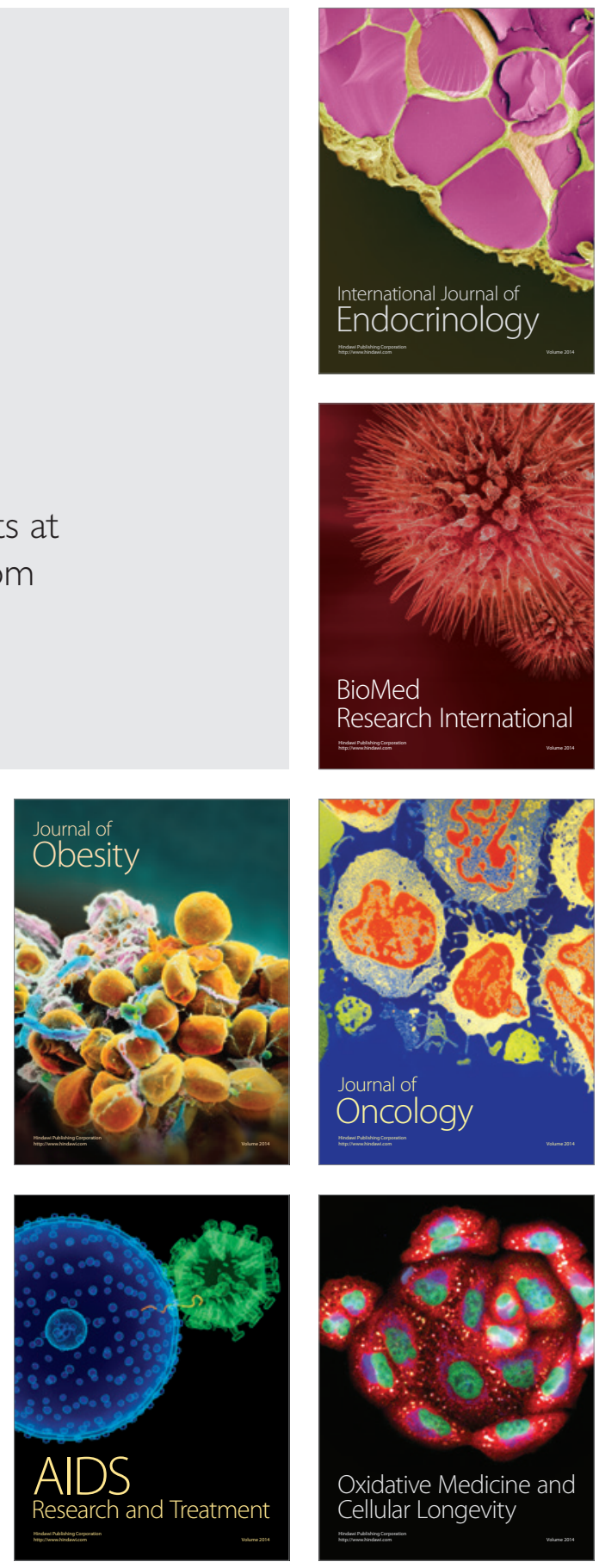